\title{
Fusion of Multiple Cue Detectors for Automatic Sports Video Annotation
}

\author{
Josef Kittler, Marco Ballette, W. J. Christmas, \\ Edward Jaser, and Kieron Messer \\ Centre for Vision \\ Speech and Signal Processing, University of Surrey \\ Guildford, Surrey GU2 7XH, UK \\ J.Kittler@eim.surrey.ac.uk
}

\begin{abstract}
This paper describes an aspect of a developing system named ASSAVID which will provide an automatic and semantic annotation of sports video. This annotation process segments the sports video into semantic categories (e.g. type of sport) and permits the user to formulate queries to retrieve events that are significant to that particular sport (e.g. goal, foul). The system relies upon the concept of "cues" which attach semantic meaning to low-level features computed on the video. In this paper we adopt the multiple classifier system approach to fusing the outputs of multiple cue detectors using Behaviour Knowledge Space fusion. Using this technique, unknown sports video can be classified into the type of sport being played. Experimental results on sports video provided by the BBC demonstrate that this method is working well.
\end{abstract}

\section{Introduction}

There is a vast amount of sports footage being recorded every day. For example, each year the British Broadcasting Corporation (BBC) provides coverage of the Wimbledon tennis championships. During this event up to fifteen different live feeds are being recorded simultaneously. At the end of the Wimbledon fortnight over one thousand tapes of sports related footage are brought back to the BBC headquarters. All this video data is generated from just one event. The BBC records hundreds of different sporting events each year.

Ideally, all this sports video should be annotated and the meta-data generated on it should be stored in a database along with the video data. Such a system would allow an operator to retrieve any shot or important event within a shot at a later date. Also, the level of annotation provided by the system should be adequate to facilitate simple text-based queries. For example a typical query could be: "Retrieve the winning shot played in the last game of each set in the ladies final in the Wimbledon tennis championship 2001". Such a system has many uses, such as in the production of television sport programmes and documentaries.

Due to the large amount of material being generated, manual annotation is both impractical and very expensive. However, automatic annotation is a very 
demanding and an extremely challenging computer vision task as it involves high-level scene interpretation. It is unlikely that an efficient, fully automated video annotation system will be realised in the near future.

Perhaps the most well known automatic video annotation system reported in the literature is Virage [1]. Virage has an open framework which allows for the integration of many real time audio and video analysis tools and places the data into an industry-standard database such as Informix or Oracle. However, the number of analysis tools available is limited, although always expanding. The main problem with Virage is that no effort has been made to bridge the gap between the information provided by the low-level analysis tools and the high-level interpretation of the video, which is required for our application.

Other work, specific to some form of sports annotation includes [9] in which camera motion is used to help in the automatic annotation of basketball. Mo et al. utilize state transition models, which include both top-down and bottom-up processes, to recognise different objects in sports scenes [8]. In [10] work has been undertaken to distinguish between sports and non-sports MPEG compressed video. Finally, MIT have been working on the analysis of American football video [4].

This paper describes one aspect of the development of a novel system which will provide a semantic annotation of sports video. This annotation process segments the sports video into semantic categories (e.g. type of sport) and permits the user to formulate queries to retrieve events that are significant to that particular sport (e.g. goal, foul). The system will aid an operator in the generation of the high-level annotation for incoming sports video. The basic building blocks of the system are low-level audio and video analysis tools, which we term cue detectors. Examples of cue-detectors include: grass; swimming pool lanes; ocean; sprint frequency; referee whistle and crowd cheer. A contextual reasoning engine will then be used to analyse the output of these cue detectors and attach semantic information to the video being analysed. The generated meta-data and video data will then be stored in a database which is based on a mixture of IBM's Media360 and Informix. The system will also provide a Java graphical user interface to the database which will allow the user to browse the video, view sequences and generate story boards, formulate queries and analyse and modify the generated indices.

In general, different methods can be adopted to design cue detectors. For instance, the same cue can be detected on the basis of different image properties, such as texture, colour or shape. Having, for a given cue, more than one cue detector raises the problem of how the respective response of these multiple detectors should be combined to improve the confidence in the cue being present or absent.

In this paper we adopt the multiple classifier system approach to fusing the outputs of multiple cue detectors. We use the Behaviour Knowledge Space fusion method [3] and show that significant improvements can be gained in this way.

The paper is organised as follows. In Section 2 we briefly describe the cue detectors used in this study. The fusion technique is overviewed in Section 3. 
The results of the experiments designed to demonstrate the effectiveness of the method are presented in Section 4 and 5. The paper is concluded in Section 6.

\section{Cue Detectors}

The objective in the automatic annotation of video material is to provide indexing material that describes as usefully as possible the material itself. In much of the previous work in this area (for example [2]), the annotation consisted of the output of various feature detectors. By itself, this information bears no semantic connection to the actual scene content - it is simply the output of some image processing algorithms. In this project we are taking the process one stage further. By means of a set of training processes, we aim to generate an association between the feature detector outputs and the occurrence of actual scene features. Thus for example we might train the system to associate the output of a texture feature detector with crowds of people in the scene. We can then use this mechanism to generate confidence values for the presence of a crowd in a scene, based on the scene texture. We denote the output of this process as a "cue". These cues can then be combined in the contextual reasoning engine to generate higher-level information, e.g. the type of sport being played.

We have developed many different cue detection methods. In this section we briefly discuss three visual cue generation methods. Each method can be used to form a number of different cue-detectors provided that suitable training data is available. These methods are neural network, multimodal neighbourhood signature and texture codes. These are briefly described in the following subsections.

\subsection{Neural Network}

Each cue-detector is a neural network trained on colour and texture descriptors computed at a pixel level on examples image regions of the cue of interest (see [7]) and on image regions which are known not to contain the cue. The resulting trained network is then able to distinguish between the features of cue and noncue pixels. A high output represents the case when the feature vector of the pixel belongs to same distribution as the cue and vice-versa.

To check for the presence of a cue in a test image the same colour and texture features are computed for each test image pixel and the feature vector is passed to the neural network. If many high outputs are observed then this gives an indication of how likely it is that the given cue is present in the image. Cues suitable for this method include sky, grass, tennis court and athletics track.

\subsection{Multimodal Neighbourhood Signature}

In the Multimodal Neighbourhood Signature (MNS) approach (described in detail in [6]), object colour structure is represented by a set of invariant features computed from image neighbourhoods with a multimodal colour density function. The method is image-based - the representation is computed from a set of examples of the object of interest. 
In the implemented method, an MNS is a set of invariant colour pairs corresponding to pairs of coordinates of the located density function modes from each neighbourhood. The MNS's of all the example images are then merged into a composite one by superposing the features (colour pairs). Considering each colour pair as an independent object descriptor (a detector), its discriminative ability is measured on a pre-selected training set consisting of positive and negative example image regions. A simple measure, the absolute difference of true and false positive percentages is computed. Finally, the $n$ most discriminative detectors are selected to represent the object of interest. For the reported experiments $n$ was set to 3 .

For matching, we view each detector as a point in the detector space. A hypersphere with radius $h$ is defined around each point. Given another image of the object, measurements are likely to lie inside the detector hyper-spheres. A binary $n$-tuple is computed for each test image, each binary digit assigned 1 if at least one test measurement was within the corresponding detector sphere, 0 otherwise. One of $2^{n}$ possible $n$-tuples are the measurements output from the matching stage. The relative frequency of each possible $n$-tuple over the positive and negative cue examples of the training set define an estimate of the probability of each measurement given the cue and not given the cue respectively. These 2 numbers are output to the decision making module.

\subsection{Texture Codes}

The texture-based cue detector consists of two components: a training phase, in which a model for the cue is created using ground truth, and the cue extractor (see [5]). In the training stage, template regions from the keyframes are selected for each cue. Several templates are needed for each cue to account for appearance variations. Textural descriptors are extracted form the templates using a texture analysis module based on Gabor filters. These descriptors, with the number of occurrences, form the model for the cue.

In the cue extractor, the whole image is presented to the texture analysis module. Then, by comparing the result with the model, a coarse detection component selects the three templates which are most likely to be visually similar to an area of the image being annotated. The similarity is evaluated using the histogram intersection. We increase the computational efficiency by hashing the meta-data; this also enables us to compute the similarity measure only for templates which share descriptors with the input image. A localisation component finally identifies the areas of the image which the selected templates match most closely, and the image location which yields the best match confidence is retained. The highest confidence, with its location, are the output for the cue.

\section{Fusion Method}

The fusion method we experimented with is the Behaviour Knowledge Space [3] which proved to be very effective in other applications. In order to describe it, let 
us first introduce the necessary notation. We consider the task of cue detection as a two class pattern recognition problem where pattern $\mathrm{Z}$ (video key frame, video sequence, audio segment) is to be assigned to one of the 2 possible classes $\omega_{i} i=1,2$. Let us assume that we have L classifiers, each representing the given pattern by some measurement vector. Denote the measurement vector used by the $j^{\text {th }}$ classifier $\mathbf{x}_{j}$. The measurement vectors may be distinct, as, for example, in the case of the multimodal neighbourhood signature expert, or some components may be identical (shared) as exemplified by the texture codes and neural network experts. Each classifier computes the respective aposteriori probabilities for the two hypotheses that a cue is either present or absent. The aposteriori probability for the cue being present, computed by expert $j$ will be denoted $P\left(\omega_{1} \mid \mathbf{x}_{j}\right)$. The probability that the cue is absent $P\left(\omega_{2} \mid \mathbf{x}_{j}\right)$ is given by $1-P\left(\omega_{1} \mid \mathbf{x}_{j}\right)$.

The Behaviour-Knowledge Space (BKS) method proposed by Huang et al [3] also considers the support from all the experts to all the classes jointly. However, the degree of support is quantified differently than in the Decision Templates approach. Here the decisions $\Delta_{j i}$ of experts $i=1, \ldots, L$ regarding the class membership $\omega_{j}, j=1, \ldots, c$ of pattern $Z$ are mapped into an $L$ dimensional discrete space and the BKS fusion rule is defined in this space. In order to be more specific, let us designate the decision of the $j^{\text {th }}$ expert about pattern $Z$ by $\delta_{j}(Z)$ which can be expressed as

$$
\delta_{j}(Z)=\operatorname{argmax}_{i=1}^{c} P\left(\omega_{i} \mid \mathbf{x}_{j}\right)
$$

Thus $\delta_{j}(Z)$ assumes integer values from the interval $[1, c]$. The combination of the decision outputs $\delta_{j}(Z), j=1, \ldots, L$ defines a point in the $L$-dimensional discrete space, referred to as the Behaviour Knowledge Space (BKS). We can consider each point in this space to index a bin (cell). The BKS fusion rule then associates a separate consensus decision with each of the bins in the BKS space.

Let us denote by $h_{i}\left(d_{1}, . ., d_{L}\right)$ the histogram of the patterns from a set $\Xi$ which belong to class $\omega_{i}$ and fall into the bin $\left(d_{1}, . ., d_{L}\right)$ by virtue of the indexation process defined in (1). The BKS fusion rule then assigns a new pattern $Z$ as follows

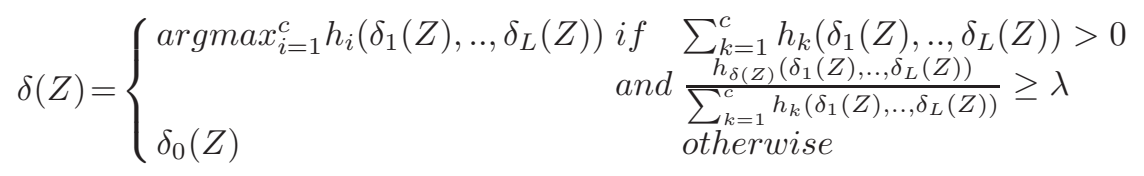

where $\delta_{0}(Z)$ denotes a rejection or a decision by an alternative fusion process such as the majority Vote, or the fusion strategies suggested in [11] and [3]. Thus a special line of action is taken when the indexed bin of the BKS histogram is zero, or if the proportional vote held by the most representative class in the bin is below threshold $\lambda$. In our two class experiments the above conditions were always satisfied. However, a number of studies on how to find the value of the threshold have been reported (see for instance Huang and Suen [3].

In summary, the class with the greatest number of votes in each bin is chosen by the BKS method. In our experiments we considered different weights for the 
two different classes based on the class a priori probabilities. Thus for each combination of classifiers we divided the number of occurrences of each class by the respective numbers of samples in set $\Xi$.

\section{Experiments}

The aim of the experiments described in this section was to investigate the effect of multiple expert fusion in the context of sports video cue detection. We considered the following sports: boxing, shooting, swimming and tennis. In each frame we looked for a boxing ring, a shooting target, a swimming pool and a tennis court. These cues are considered indicative of the respective sport disciplines. The images in figure ?? show such examples for each sport and cue. The study was limited to these four cues because for each of them we had the responses from multiple cue detectors that we wished to combine. In particular, for each cue we had the outputs of three experts. These experts and the associated identifiers (codes) are : Texture Code Expert (code 0 ), Multimodal Neighbourhood Signature Expert (code 1), Neural Net Expert (code 2).

The experiments were conducted on a database of key frames which were manually annotated to provide ground truth information about cue presence. The database contained 517 key frames of boxing, 172 frames of shooting, 1087 frames of swimming and 469 frames of tennis. Each key frame then had the cueoutputs for each cue-detector and each cue-method (expert) computed. Thus for each expert we had $517^{*} 4$ outputs for boxing, $172^{*} 4$ outputs for shooting and so on. Each detector generated two scores : $p\left(\mathbf{x}_{j} \mid \omega_{1}\right)$ and $p\left(\mathbf{x}_{j} \mid \omega_{2}\right)$. These scores are the density function values for the key frame measurements $\mathbf{x}_{j}$ computed by the $\mathrm{j}^{\text {th }}$ cue-detector when the cue is present and absent from the scene respectively. These scores are converted into aposteriori class probabilities under the assumption that, a priori, the presence and absence of a cue are equally likely, i.e.

$$
P\left(\omega_{1} \mid \mathbf{x}_{j}\right)=\frac{p\left(\mathbf{x}_{j} \mid \omega_{1}\right)}{p\left(\mathbf{x}_{j} \mid \omega_{1}\right)+p\left(\mathbf{x}_{j} \mid \omega_{2}\right)}
$$

A global thresholding was then applied in order to obtain a crisp label.

We split our data set into an evaluation set and a test set. We considered two different configurations that we called CONFIGURATION 1 (evaluation set $=$ $20 \%$ of the total set; test set $=80 \%$ of the total set) and CONFIGURATION 2 (evaluation set $=30 \%$; test set $=70 \%$ ). The evaluation set was used to find the optimal global threshold which produced the lowest total error rate. The performance of the fusion method was then evaluated on a completely independent test set.

The results of the experiments are presented in Figures ??(a) and ??(b). The false rejection and false acceptance error rates of the system were estimated separately and then averaged. This is a standard practice in detection problems as it is impossible to specify the prior probabilities of the populations of key frames which do and do not contain a particular cue accurately. The resulting error rates are then shown in the figures for the four sport disciplines. 
In general, the error rates are lower for CONFIGURATION 2 than for CONFIGURATION 1. This is understandable, as CONFIGURATION 2 uses more data for training the fusion system than CONFIGURATION 1.

Boxing In the case of boxing cue detection it is interesting to note that the best pair of experts, as selected on the evaluation set, did not include the individually best cue detector based on MNS. This is reflected in the performance on the test set which is worse than that of the individually best detector. Once the size of the evaluation set is increased, better generalisation behaviour is observed. This is apparent from the monotonicity of the fusion results, i.e. as the number of detectors is increasing, the performance monotonically improves.

Shooting In the case of the shooting cue, the Texture Code and Neural Network detectors produced very high false acceptance rates. Interestingly, the MNS detector has a zero rejection rate as the shooting cue - shooting target - is a very distinctive object. There was a dramatic swing of false acceptance and false rejection rates between the two configurations. Again, CONFIGURATION 2 results exhibited better generalisation and most importantly, the benefit of multiple detector fusion was the most pronounced.

Swimming For CONFIGURATION 1, both texture codes and MNS detectors produced very high false rejection rates and all detectors showed zero false acceptance rate. The imbalance in performance was corrected, though with some overshoot, with the enhanced training under CONFIGURATION 2. Again, much better generalisation was achieved for CONFIGURATION 2.

Tennis The performance trends noted in the case of tennis were quite consistent with the previous cues. The main points are the improved generalisation when moving from CONFIGURATION 1 to CONFIGURATION 2. Most importantly, the multiple cue detector fusion consistently provides at least as good or better results than the individually best detector.

\section{Results on a Relabelled Database}

A detailed analysis of the frequently unbalanced error rates and the difficulty in selecting a sensible threshold reported in the previous section revealed that the problem derived primarily from the way the data set was labelled. Any key frame that was part of a video segment reporting on a particular sport was routinely assigned the label corresponding to that sport. Yet the individual cue detectors were trained to detect specific cues that are characteristic of the respective disciplines. For instance, the visual content of the swimming cue was an image segment of the swimming pool containing ropes delineating the swimming lanes. However, some segments of the swimming video sequence contained interviews with the competitors and the swimming pool was not visible. Often the number of such frames was significantly high and this resulted in a complete overlap of the cue present and cue absent distributions. In view of this, all the key frames were carefully re-examined and whenever appropriate re-labelled.

The experiments of the previous section were then repeated. The results are reported in Figures ??(a) and ??(b) using the same format of presentation. In 
general, we observe that the error rates are more balanced, although not always better than in the previous section. However, most importantly we observe dramatic improvements in the results of the multiple cue detector system for all disciplines. The results are particularly promising for CONFIGURATION 2 for which average error rates are not worse than $3.11 \%$. In the case of shooting they drop to zero both for false rejection and false acceptance.

\section{Conclusion}

In this paper we have described a process for the automatic sports classification within the developing ASSAVID system. We have demonstrated that the method, based on the concept of cue detection, is working well on a set of ground-truthed static images. It has also been demonstrated that by adopting the multiple classifier system approach of Behaviour Knowledge Space to fusing the outputs of the multiple cue detectors can significantly improve the performance of the automatic sports classification.

It was also demonstrated that the sports were only recognised when a suitably trained cue for that sport was identified in the image. If that cue for that sport was not in the image the frame was incorrectly labelled. The use of more cues for each specific sport should increase the recognition accuracy and robustness. At present we are working on more cue-methods and training more cue-detectors. These include cues based on other audio and motion features.

In the final system it is intended to make a decision about the sport being played over an entire shot and not just a single frame. This will allow us to incorporate temporal information into the decision making decision and this should robustify the results further.

\section{Acknowledgements}

This work has been performed within the framework of the ASSAVID project granted by the European IST Programme.

\section{References}

1. http://www.virage.com. 598

2. B. V. Levienaise-Obadia, W. Christmas, J. Kittler, K. Messer, and Y. Yusoff. Ovid: towards object-based video retrieval. In Proceedings of Storage and Retrieval for Video and Image Databases VIII (part of the SPIE/ITT Symposium: Electronic Imaging'2000), Jan 2000. 599

3. Y. Huang and C. Suen. A method of combining multiple experts for the recognition of unconstrained handwritten numerals. IEEE Transaction on Pattern Analysis and Machine Intelligence, 17(1), 1 1995. 598, 600, 601

4. S. S. Intille and A. F. Bobick. A framework for representing multi-agent action from visual evidence. In Proceedings of the National Conference on Artificial Intelligence (AAAI), July 1999. 598 
5. B. Levienaise-Obadia, J. Kittler, and W. Christmas. Defining quantisation strategies and a perceptual similarity measure for texture-based annotation and retrieval. In IEEE, editor, ICPR'2000, volume III, Sep 2000. 600

6. J. Matas, D. Koubaroulis, and J. Kittler. Colour Image Retrieval and Object Recognition Using the Multimodal Neighbourhood Signature. In D. Vernon, editor, Proceedings of the European Conference on Computer Vision, LNCS vol. 1842, pages 48-64, Berlin, Germany, June 2000. Springer. 599

7. K. Messer and J. Kittler. A region-based image database system using colour and texture. Pattern Recognition Letters, pages 1323-1330, November 1999. 599

8. H. Mo, S. Satoh, and M. Sakauchi. A study of image recognition using similarity retrieval. In First International Conference on Visual Information Systems (Visual'96), pages 136-141, 1996. 598

9. D. D. Saur, Y.-P. Tan, S. R. Kulkarni, and P. J. Ramadge. Automated analysis and annotation of basketball video. In SPIE Storage and Retrieval for Still Image and Video Databases V, Vol.3022, pages 176-187, 1997. 598

10. V. Kobla, D. DeMenthon, and D. Doermann. Identifying sporst video using replay, text and camera motion features. In SPIE Storage and retrieval for Media Database 2000, pages 332-342, 2000. 598

11. K.-D. Wernecke. A coupling procedure for the discrimination of mixed data. Biometrics, 48:497-506, 6 1992. 601

12. L. Xu, A. Krzyzak, and C. Y. Suen. Methods of combining multiple classifiers and their applications to handwriting recognition. IEEE Transaction. SMC, 22(3):418435, 1992. 